\title{
Study on Electronic Differential Control System Used for In-wheel Motor Driven EV
}

\author{
Chuanwei Zhang, Yue Yuan, Nuoting Wang , Chenxi Li \\ Xi'an University of Science And Technology , Xi'An,China \\ 395756524@qq.com
}

Keywords:Speed characteristics ; Electric vehicle ; Electronic differential ;

Abstract:In view of the features of In-wheel Motor Driven EV, Ackermann steering differential model was established by using MATLAB/Simulink. Based on this model, the features have been studied under different steering angle and velocity of four wheels in this paper. The feasibility of differential algorithm was validated by the simulation results .

\section{Introduction}

Energy shortage and environmental pollution seriously restricts the development of social economy. Energy saving and environmental protection has become the focus around the world. In order to alleviate environmental and energy problems, electric vehicles, as the focus of the future automotive industry, entered a period of rapid development ${ }^{[1,2]}$. China attaches the great importance to the development of electric vehicle technology, which was designated as a pillar industry of Chinese industry. At the same time, the ministry of science and technology for the development of electric vehicles proposed several major projects, planning the future prospects of the development of the electric vehicles and the technical route of future electric vehicles ${ }^{[3]}$.

The driving wheel speed is different in the process of moving under the cornering or uneven road conditions. Conventional vehicles' differential can be achieved through the coordination of mechanical differential ${ }^{[4]}$. Via the electronic differential to achieve In-wheel motor driven electric vehicles differential control due to each wheel is independent. Based on the Ackermann steering model, the independent drive electric vehicle is controlled by electronic differential. Firstly, in this paper, the vehicle structure was set up. Secondly, based on the Ackermann steering differential model, differential control was put forward. Finally, the feasibility of the differential model was verified by the simulation analysis under different angle and speed of the wheel speed characteristics.

\section{Overall Structure}

In order to fit the requirements of in-wheel motor electric vehicle dynamics control, according to the desired requirements, the overall structure is defined, and electronic architecture is designed ${ }^{[5]}$. Electronic architecture of electric vehicle reflects the experimental platform design goals. The testing prototype hardware interface definition and all subsystems are deployed around the architecture. The vehicle electronic structure is shown in Fig.1.

Using dSPACE company MicroAutobox rapid prototyping controller as the vehicle controller to concentrate on the control algorithms. Prototype controller has a high-speed computing power, a wide range of I/O interface and function of auto signal processing. It can easily read each sensor collected information and CAN bus acquisition information. The development of software is based on the graphical interface. Eliminating the needs for developers to debug complex manual programming errors and easy to work with Matlab / Simulink, control model can be created in Matlab/Simulink by automatically generating code which can be identified and executed. The development process of control algorithm is simplified, moreover, the development efficiency is improved. 


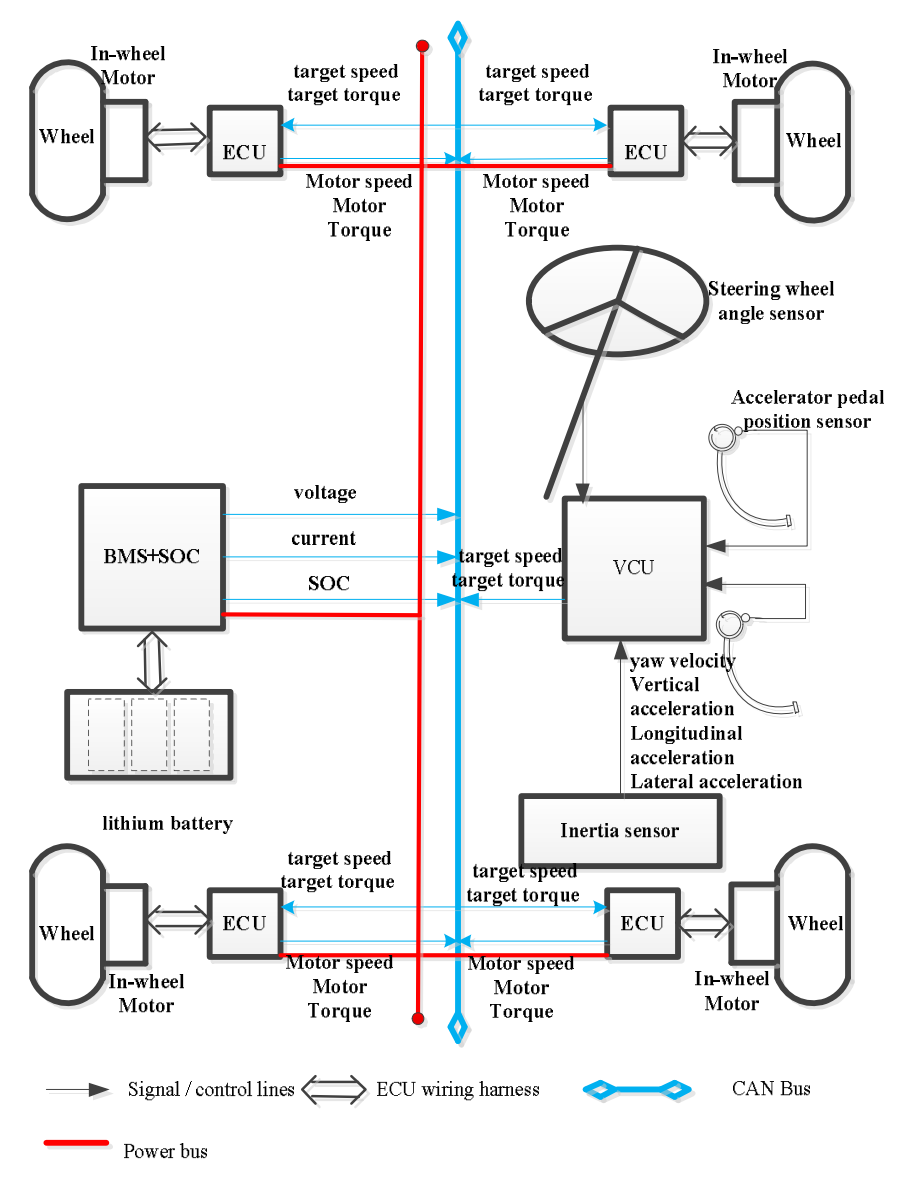

Fig. 1 The vehicle electronic structure

The machine body of the testing prototype is the frame and suspension. The four $72 \mathrm{v} / 3 \mathrm{kw}$ brushless DC in-wheel motor were installed directly in the tire, and each motor is controlled by a motor controller directly. Optional power battery is 72V/100Ah lithium iron phosphate batteries, they are used to power the vehicle support. Battery management system (BMS) can collect the battery charge and discharge status, the information such as voltage, current and battery. lithium-ion battery pack for equalizing charge prevents overcharge and discharge. Battery systems have short circuit protection, over-current protection, prolong the service life of batteries.

There are accelerator pedal position sensor, brake pedal position sensor, steering wheel Angle sensor, acceleration and yawing velocity sensor in the testing prototype .The accelerator pedal is used to determine the driver's intention, it is the key to calculate the desired longitudinal force, its output signal is analog signal, which will be used as the input variables of vehicle control. Steering wheel angle sensor is to measure the angle of the steering wheel turned. The front wheel angle can be obtained by steering wheel angle sensor. Front wheel angle is a key parameter to calculate the yaw moment. The accelerator pedal and steering wheel angle are the input parameters of the control system for vehicle.

In addition, VBOX vehicle data acquisition system is selected as the information acquisition of vehicles running state tool in this paper, it selects the acceleration, pitch, roll, yaw and other vehicle information at the same time. The position signal and the speed signal acquired are accurate. Meanwhile, in order to determine the basic parameters of the testing prototype and build prototype model of the testing prototype, basic parameters of the testing vehicle are shown in Tab1. 
Table 1 Basic parameters of the test

\begin{tabular}{ll}
\hline \multicolumn{1}{c}{ Parameter } & Technical value \\
\hline $\begin{array}{l}\text { boundary dimension } \\
\text { (length*width*height })\end{array}$ & $3600^{*} 1440 * 1780$ \\
mm & 1860 \\
wheel base $(\mathrm{mm})$ & 1210 \\
wheel track $(\mathrm{mm})$ & 4500 \\
minimum turning diamete \\
$\quad(\mathrm{mm})$
\end{tabular}

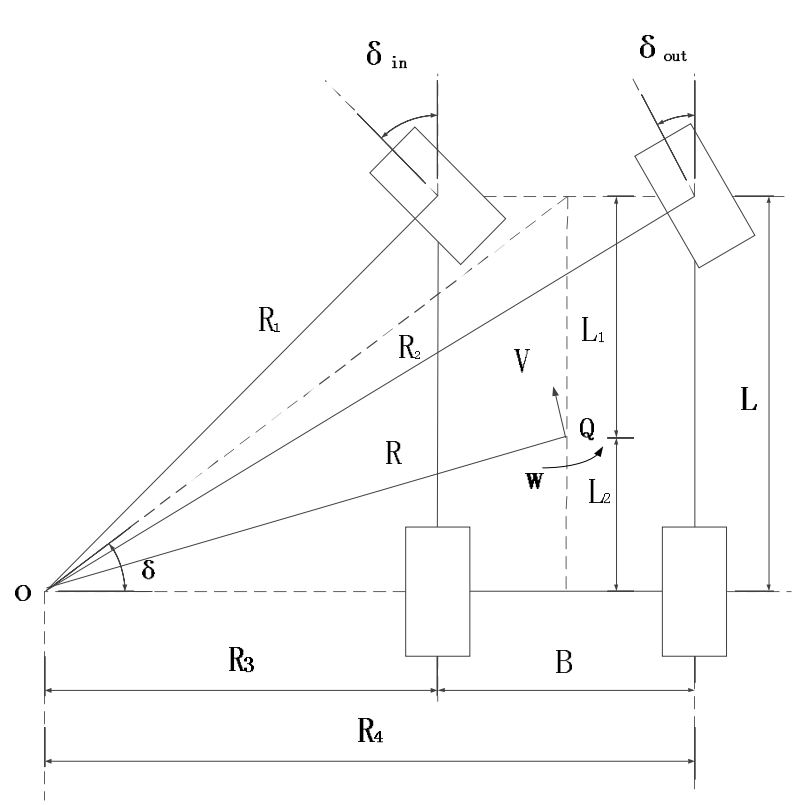

Fig.2 Ackermann steering model diagram

\section{Differential model}

Ackerman steering model ${ }^{[6]}$ is established under the low speeds when steering as shown in Fig 2, which must follow the conditions: 1) the vehicle body must be rigid; 2) wheels for pure rolling motion, without considering slip or slip conditions; 3 ) ignore the impact of changes of centrifugal force and loads of vehicle to wheels in steering.

According to Ackermann model, design of the vehicle parameters: $\mathrm{L}$ is the wheel base; $\mathrm{B}$ is the wheel tread; $L_{1}$ and $L_{2}$ respectively are the distance from front axle and the rear axle to the center of mass; $\delta$ is Ackermann steering angle; $\delta_{\text {in }}$ and $\delta_{\text {out }}$ are inside and outside front wheel steering angle; Respectively, $\delta_{\text {in }}>\delta_{\text {out }}$. When steering, the vehicle rotates around the center $\mathrm{O}$, the center $\mathrm{O}$ is collinear with the rear axle steering. $\mathrm{V}_{1}, \mathrm{~V}_{2}, \mathrm{~V}_{3}, \mathrm{~V}_{4}$ are left front wheel, right front wheel, left rear wheel, right rear wheel speeds respectively. $R_{1}, R_{2}, R_{3}$ and $R_{4}$ are the radius of the wheels around the steering center $\mathrm{O}$ respectively. $\mathrm{V}$ is the vehicle speed. $\mathrm{R}$ is the radius that is the distance between vehicle mass center $\mathrm{Q}$ and the steering center $\mathrm{O}$. According to the geometric relationship, equations are shown below:

$$
\begin{aligned}
& \tan \delta_{\text {in }}=\frac{L}{R_{3}}=\frac{L}{\frac{L}{\tan \delta}-\frac{B}{2}}=\frac{2 L \tan \delta}{2 L-B \tan \delta} \\
& \tan \delta_{\text {out }}=\frac{L}{R_{4}}=\frac{L}{\frac{L}{\tan \delta}+\frac{B}{2}}=\frac{2 L \tan \delta}{2 L+B \tan \delta}
\end{aligned}
$$




$$
\begin{aligned}
& V_{3}=\frac{V * R_{3}}{R}=V * \frac{\sqrt{\left(\frac{L}{\tan \delta}-\frac{B}{2}\right)^{2}}}{\sqrt{L_{2}^{2}+\left(\frac{L}{\tan \delta}\right)^{2}}} \\
& V_{4}=\frac{V * R_{4}}{R}=V * \frac{\sqrt{\left(\frac{L}{\tan \delta}+\frac{B}{2}\right)^{2}}}{\sqrt{L_{2}^{2}+\left(\frac{L}{\tan \delta}\right)^{2}}} \\
& R_{1}{ }^{2}=L^{2}+R_{3}{ }^{2}=L^{2}+\left(\frac{L}{\tan \delta}-\frac{B}{2}\right)^{2} \\
& R_{2}{ }^{2}=L^{2}+R_{4}{ }^{2}=L^{2}+\left(\frac{L}{\tan \delta}+\frac{B}{2}\right)^{2}
\end{aligned}
$$

By the instantaneous center theorem:

$$
\frac{V}{R}=\frac{V_{1}}{R_{1}}=\frac{V_{2}}{R_{2}}=\frac{V_{3}}{R_{3}}=\frac{V_{4}}{R_{4}}
$$

Too :

$$
\begin{aligned}
& \frac{V_{1}}{V_{2}}=\frac{R_{1}}{R_{2}}=\frac{\sqrt{L^{2}+\left(\frac{L}{\tan \delta}-\frac{B}{2}\right)^{2}}}{\sqrt{L^{2}+\left(\frac{L}{\tan \delta}+\frac{B}{2}\right)^{2}}} \\
& V_{1}=\frac{V * R_{1}}{R}=V * \frac{\sqrt{L^{2}+\left(\frac{L}{\tan \delta}-\frac{B}{2}\right)^{2}}}{\sqrt{L_{2}{ }^{2}+\left(\frac{L}{\tan \delta}\right)^{2}}} \\
& V_{2}=\frac{V * R_{2}}{R}=V * \frac{\sqrt{L^{2}+\left(\frac{L}{\tan \delta}+\frac{B}{2}\right)^{2}}}{\sqrt{L_{2}{ }^{2}+\left(\frac{L}{\tan \delta}\right)^{2}}}
\end{aligned}
$$

According to the established differential relationship, the wheel speed ratio of the steering wheel has no relation with speed $\mathrm{V}$, only with the steering angle and body structure parameter, wheel base $\mathrm{L}$, wheel track $\mathrm{B}$, the distance $\mathrm{L}_{2}$ from center of mass to the rear axle; Each wheel speed is related with the actual vehicle speed $\mathrm{V}$, Ackerman steering angle $\delta$ and the vehicle body structure parameters wheel base L, wheel track B. 


\section{Simulation Modeling and Analysis}

According to the calculation formula of the Ackermann steering model, using the Matlab/Simulink to establish the differential prototype model. The model inputs are the steering angle, practical speed Vand the structural parameters of vehicle model, the output is the different wheel speed.

To verify the feasibility of the differential algorithm: validated by changing the single variable, the 4 different speeds were applied which are $4 \mathrm{~m} / \mathrm{s}, 8 \mathrm{~m} / \mathrm{s}, 12 \mathrm{~m} / \mathrm{s}$ and $16 \mathrm{~m} / \mathrm{s}$. When the left steering is processing the steering angle is required to increase, the speed characteristic of each wheel was analyzed. The simulation results are shown in Fig3 to Fig. 6:

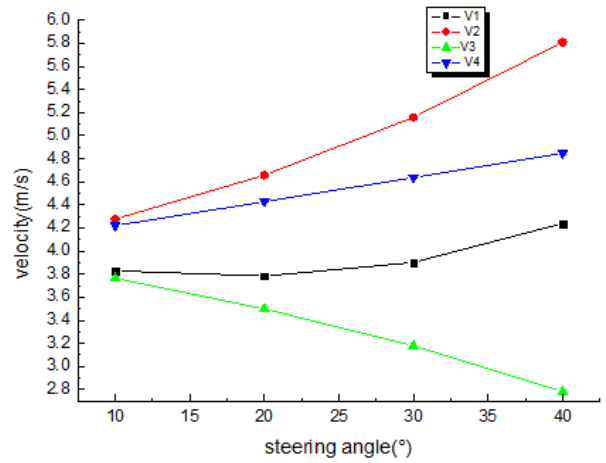

Fig.3 dbiagram etween each wheel speed and the steering angle when velocitv is $4 \mathrm{~m} / \mathrm{s}$

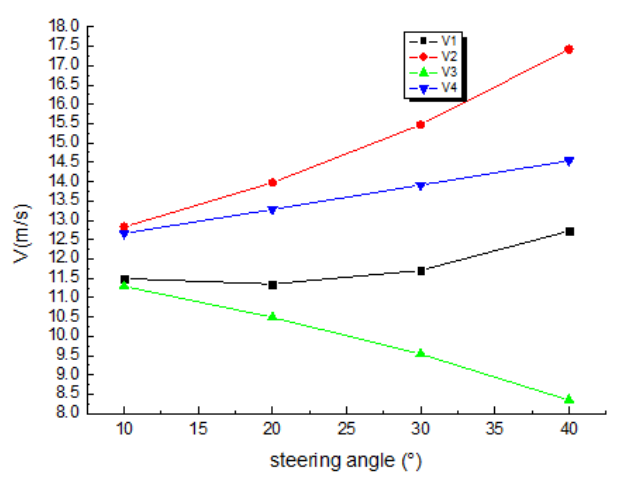

Fig.5 diagram between each wheel speed and the steering angle when velocitv is $12 \mathrm{~m} / \mathrm{s}$

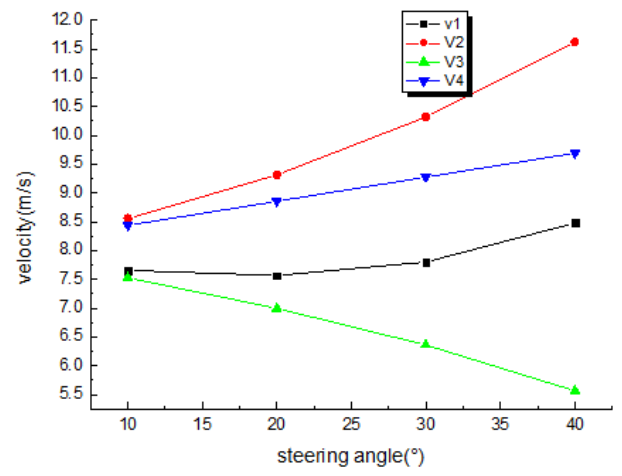

Fig.4 diagram between each wheel speed and the steering angle when velocitv is $8 \mathrm{~m} / \mathrm{s}$

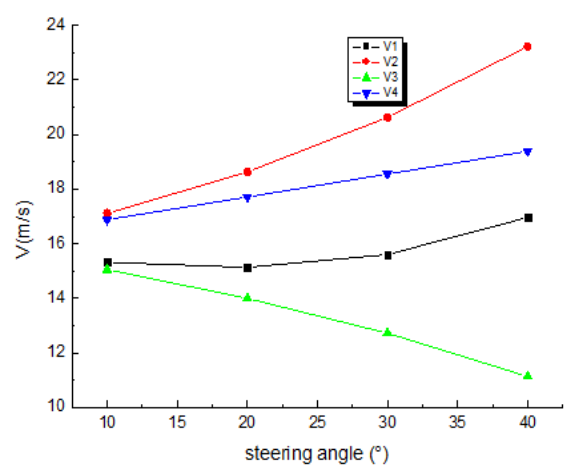

Fig.6 diagram between each wheel speed and the steering angle when velocitv is $16 \mathrm{~m} / \mathrm{s}$

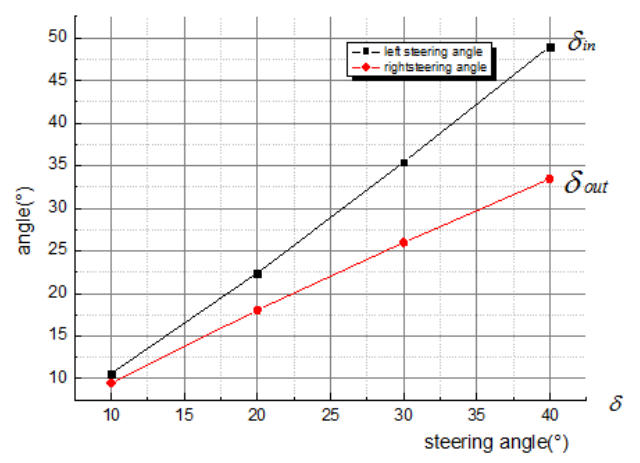

Fig.7 diagram between the inner and outer front wheel steering angle

Known from the analysis of the above simulation data: when the vehicle is turning, the wheel speed of the outer wheel is always higher than the inner's, it's not affected by the steering angle and the actual speed; The inside steering wheel speed increases with the angle changed in small doses; the inner wheel speed changes little with the increase of steering angle; With the increase of the steering angle, the outer front wheel speed increases quickly, the inner rear wheel speed decreases faster. Therefore, when the understeer or oversteer 
situation is happening, the most useful way to achieve differential is changing inner rear wheel or outer front wheel; With the actual vehicle speed increasing, the speed of each wheel is increased; With the increase of steering angle, the inner steering angle increases faster than the outer, the result can be gained from Fig. 7.

Differential model is established in this paper which is also simplified according to the actual process of the steering wheel differential case. It ignores the impact of tires, road surface and the vertical load to steering wheel. Although there are small quantities of differences with the actual wheel speed, it does not affect the theoretical analysis. This model is suitable for low-speed steering. The maximum speed of the experimental prototype is limited to $15.3 \mathrm{~m} / \mathrm{s}$. In fact, when the vehicle is turning, the outer wheel speed will not increase until $15.3 \mathrm{~m} / \mathrm{s}$ and keep it. With the steering angle and vehicle speed increasing, each wheel is kept at the maximum speed. The simulation data of Fig 6. shows that the outer wheel speed is more than 15.3 $\mathrm{m} / \mathrm{s}$, other wheel speed also changes accordingly. In other words, this differential model is not able to fit the practical requirements, moreover, it shows the limitations of the differential model.

\section{Conclusion}

As for in-wheel motor driven EV, Ackermann differential algorithm has been studied which fits the low steering. Based on the model of the differential which was established already, each wheel speed in electric vehicles under different actual speeds and steering angle are obtained. The simulation shows the speed characteristics of four wheel of pure electric vehicle under different speed and steering angle. Through analyzing, the Ackermann differential algorithm fully fit the requirements of the testing prototype, and provide a reliable theoretical basis for the differential control. This algorithm is simple but practically useful, it can realize differential demands of the vehicle within $50 \mathrm{~km} / \mathrm{h}$ steering speed.

In addition, in order to be more efficient and more accurate, control strategy based on the Ackermann steering model electronic differential control and hierarchical control torque coordination will be adopted in the further study. Torque difference between inner driving wheel and outer driving wheel will be calculated by driver's steering wheel angle, vehicle speed and feedback parameters dynamic model will be given by itself. Then the driving torque will be distributed according to the driver's desired driving torque, the inner and outer wheels will generate different speeds, thus electronic differential will be very likely achieved.

\section{References :}

[1] SPERLING D, GORDON D. Two billion cars: driving toward sustainability[M]. New York: Oxford University Press, 2009.

[2] MITCHELL W J, BORRONI-BIRD C E, BURNS L D. Reinventing the automobile: personal urban mobility for the 21st century[M]. Cambridge: The MIT Press, 2010.

[3] " Twelfth five-year " special plan of Electric vehicle technology development, Ministry of Science and Technology of the People's Republic of China, 2012,In Chinese.

[4] Yuan Yi, Chen Shiyuan, Liu Yaoge. Summary of electric vehicles electronic differential Solutions [J]. Shanghai Automotive, 2009 (3) : 1-5, In Chinese.

[5] Bai Hongtao. Study on drive control strategy used for four-wheel-hub motor pure electric vehicle [D]. Jilin University, 2015, In Chinese. 
[6] ACKERMANN J, ODENTHAL D, BUNTE T. Advantages of active steering for vehicle dynamics control[C]// Vienna 32nd International Symposium on Automotive Technology and Automation, 1999. 